\title{
Structure of an Experimentally Evolved Gene Duplication Encoding Ribitol Dehydrogenase in a Mutant of Klebsiella aerogenes
}

\author{
By MICHAEL S. NEUBERGER*† AND BRIAN S. HARTLEY \\ Department of Biochemistry, Imperial College of Science and Technology, \\ London SW7 $2 A Z$
}

(Received 16 June 1980)

\begin{abstract}
We have previously described a system of experimental evolution in which many of the mutants of Klebsiella aerogenes selected for faster growth on xylitol ('evolvants') synthesized elevated levels of ribitol dehydrogenase and have presented genetic evidence implicating gene duplication in the enzyme superproduction in some of the evolvants. Here we describe a physical approach to the screening for gene duplications and subsequent structure determination. Nick-translated, cloned ribitol operon $(r b t)$ DNA was used as a hybridization probe to identify fragments containing $r b t$ operon sequences in restriction digests of total bacterial DNA. Whilst several of the evolvants probably harbour duplications spanning the entire rbt operon, one of the spontaneously arising evolvants (strain A3) was shown to harbour a small (5.8 kilobase pairs) direct DNA repeat which encodes the dehydrogenase (but not the kinase) of the closely linked D-arabitol operon as well as the dehydrogenase (but not the kinase) of the rbt operon. The hybridization data suggest that there are 4 to 5 copies of the repeat arranged contiguously on the chromosome. The genetic instability of strain A3, the $r b t$ fragment hybridization pattern of an A3 segregant and the activities of the pentitol catabolic enzymes in A3 are all consistent with the proposed gene duplication structure.
\end{abstract}

\section{INTRODUCTION}

The evolutionary importance of gene duplication has been stressed by many authors (Lewis, 1951; Smithies et al., 1962; Ohno, 1970; Riley et al., 1978). In order to glean information about the structure and mechanism of formation of primary gene duplications, several groups have studied bacterial duplication mutants (for a review, see Anderson \& Roth, 1977). In most of these studies, duplications were identified by their segregational instability, genetic techniques being used to probe their structures. In many of the systems, the duplications were probably created by unequal crossing over between pre-existing repeated sequences present on the bacterial genome, as the $\operatorname{rec} A$ function was implicated in duplication formation and the duplications showed distinct non-randomness in the location of their end-points (Straus \& Hoffmann, 1975; Hill et al., 1977; Anderson \& Roth, 1978). However, a recA-independent mechanism of duplication generation must also exist, since the frequency of occurrence of Escherichia coli mutants carrying duplications of the arg genes is not significantly affected by lesions in the recA gene (Beeftinck et al., 1974). Furthermore, studies on mutants of phage $\lambda$ containing tandemly repeated DNA revealed that the duplication end-points were randomly distributed over the $\lambda$ genome and the frequency with which these mutants arose was unaffected by lesions in the host red or phage red and int recombination systems (Emmons \& Thomas, 1975).

The work reported here describes the analysis of mutants obtained in a system of directed evolution in which a strain of Klebsiella aerogenes constitutive for ribitol operon $(r b t)$ expression was grown in xylitol-limited continuous culture (Rigby et al., 1974). The first step of

+ Present address: MRC Laboratory of Molecular Biology, Hills Road, Cambridge CB2 2QH. 
xylitol catabolism is catalysed by ribitol dehydrogenase and, as ribitol dehydrogenase has a very low affinity for xylitol, the activity of this enzyme limits the growth rate of the organism. Mutants growing faster on xylitol ('evolvants') either synthesized elevated levels of wild-type ribitol dehydrogenase or synthesized a mutant enzyme with improved affinity for xylitol (Hartley et al., 1976). We have previously presented genetic evidence implicating gene duplication as being responsible - at least in part - for the enzyme superproduction in some of the evolvants (Rigby et al., 1974). However, a genetic analysis does not readily yield detailed information on duplication structure and, in particular, cannot be used to determine whether a specific duplication is strictly tandem. Furthermore, screening enzyme superproducers for segregational instability will only reveal gene duplications where the DNA repeats are oriented in the same direction and are not separated by essential genes. Invert duplications can occur in bacteria (Trowsdale \& Anagnostopoulos, 1975; Bergquist \& Jamieson, 1977; Charlier et al., 1979) but might not, in general, be readily detected genetically. We therefore preferred to carry out a physical screening and analysis of the gene duplications, which is described below.

\section{METHODS}

Phage and bacterial strains. Phage $\lambda$ prbt was constructed by the $\lambda$ secondary site attachment technique, using a strain of $E$. coli $\mathrm{K} 12$ that contains the $K$. aerogenes $r b t$ and dal genes; the phage encodes the ribitol dehydrogenase and D-ribulokinase activities of the rbt operon, but neither of the activities of the dal operon (Neuberger \& Hartley, 1979). All the $K$. aerogenes evolvants are ultimately descended from $K$. aerogenes 1033 strain XI (arg gua rbt-constitutive) of Wu et al. (1968). The detailed genealogy of the evolvants is presented in Fig. 1. The frequency with which evolvants segregated derivatives growing more slowly on xylitol was determined as described by Rigby et al. (1974).

Enzyme specific activities. The preparation of cell-free extracts and their assays for ribitol dehydrogenase and D-arabitol dehydrogenase have been described previously (Neuberger et al., 1979); one unit of dehydrogenase activity catalyses the reduction of $1 \mu \mathrm{mol} \mathrm{NAD}+\min ^{-1}$ under these assay conditions. D-Ribulokinase activity was measured using the cysteine-carbazole reaction to monitor the rate of disappearance of free D-ribulose in a reaction mixture containing the enzyme, D-ribulose and ATP; the assay was carried out as described previously for D-xylulokinase (Neuberger \& Hartley, 1979). Protein was measured by a modified Lowry procedure (Miller, 1959) using bovine serum albumin as the standard.

Labelling DNA by nick translation. DNA was purified from phage $\lambda$ prbt and labelled in vitro using DNA polymerase I (a gift from Dr P. W. J. Rigby) and deoxyribonucleoside [ $\alpha-{ }^{32}$ P]triphosphates (The Radiochemical Centre, Amersham) as described previously (Neuberger \& Hartley, 1979). Nick-translated DNA was separated from unincorporated deoxyribonucleoside triphosphates by gel filtration in Sephadex G-50, stored at $-20^{\circ} \mathrm{C}$ and used within 1 week.

Electrophoresis of DNA and Southern blotting. Total bacterial DNA was isolated by the method of Marmur (1961), except that the final propan-2-ol purification step was omitted. For the analysis of the rbt fragment hybridization patterns of the various evolvants, $4 \mu \mathrm{g}$ bacterial DNA in $10 \mathrm{~mm}$-Tris/ $\mathrm{HCl}, 10 \mathrm{~mm}-\mathrm{MgCl}_{2}$, $10 \mathrm{mM}-\mathrm{NaCl}, 10 \mathrm{mM}$-2-mercaptoethanol, $\mathrm{pH} 7.5$ was digested with the appropriate endonuclease. (The $\mathrm{NaCl}$ concentration was adjusted to $100 \mathrm{mM}$ for digestion with Sall.) BstI was a gift from Dr C. M. Clarke, EcoRI and HindIII were obtained from the Centre for Applied Microbiology and Research, Porton Down, Wilts., SmaI was bought from Boehringer and KpnI, BgIII, SalI and $S s t I$ were gifts from Drs D. M. Glover and P. W. J. Rigby. DNA samples were subjected to electrophoresis in $22 \times 16 \times 0.6 \mathrm{~cm}$ agarose slab gels using the Tris/borate buffer system described by Greene et al. (1974). To obviate difficulties in the interpretation of the fragment hybridization patterns created by incomplete digestion of the chromosomal DNA, parallel incubations which differed only in the endonuclease:DNA ratio were analysed on the same gel. In order to provide marker fragments of known size which would be detectable by autoradiography, samples $(0.2 \mu \mathrm{g})$ of an EcoRI + BstI digest of $\lambda c I 857$ S7 DNA were placed in the outside slots of each gel. This digest gives fragments of $15 \cdot 2,5 \cdot 3,4 \cdot 4,3 \cdot 6$ (doublet), 3.3, 3.1, 2.6, 2.4, 1.7 and 1.1 kilobase (kb) (Haggerty \& Schleif, 1976); a fragment of $8.6 \mathrm{~kb}$ is also detected which is created by the joining of the $5.3 \mathrm{~kb}$ and $3.3 \mathrm{~kb}$ fragments through the $\lambda$ cohesive end sequences. Phage $\lambda$ prbt DNA hybridizes to all except the $1.7 \mathrm{~kb}$ and one of the $3.6 \mathrm{~kb}$ fragments.

After electrophoresis, the DNA was visualized by its fluorescence with ethidium bromide and photographed during illumination with ultraviolet light. The gels were then soaked for $1 \mathrm{~h}$ in $0.5 \mathrm{M}-\mathrm{NaOH}, 1.5 \mathrm{M}-\mathrm{NaCl}$ followed by $40 \mathrm{~min}$ in $3 \mathrm{M}-\mathrm{NaCl}, 1.5 \mathrm{M}$-Tris $/ \mathrm{HCl}, \mathrm{pH} 7 \cdot 5$. The DNA was allowed to transfer over a period of $20 \mathrm{~h}$ on to preboiled nitrocellulose sheets (Schleicher \& Schuell, BA85 paper) by the method of Southern (1975). The nitrocellulose sheets were then washed with $2 \times$ SSC (SSC is $0.15 \mathrm{M}-\mathrm{NaCl}, 0.015 \mathrm{M}$-trisodium citrate) and baked for $2 \mathrm{~h}$ at $80^{\circ} \mathrm{C}$. To prevent further binding of single-stranded DNA to the paper, the sheets were incubated for 


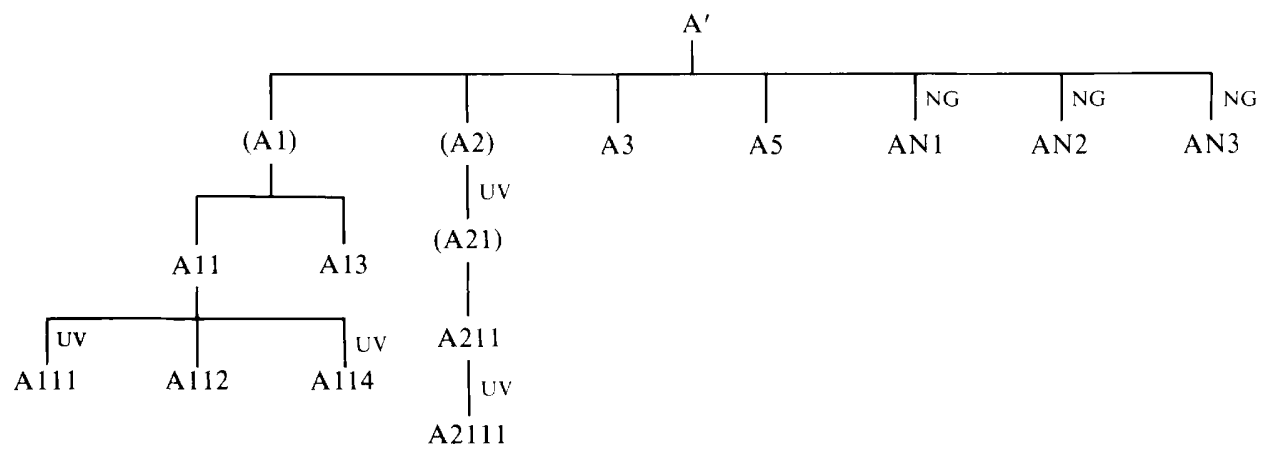

Fig. 1. Genealogy of some of the $K$. aerogenes evolvants. All evolvants were selected for fast growth in xylitol-limited continuous culture and all arose spontaneously unless otherwise indicated (Uv, ultraviolet light mutagenesis, NG, mutagenesis with $N$-methyl- $N^{\prime}$-nitro- $N$-nitrosoguanidine); all except those in parentheses were screened for $r b t$ duplications as described in the text. Genetic evidence indicates that evolvants A11 and A211 contain duplications of the wild-type ribitol dehydrogenase gene (Rigby et al., 1974) and that evolvant AN3 is duplicated for a gene encoding a mutant ribitol dehydrogenase with improved affinity for xylitol (Dothie, 1974).

$16 \mathrm{~h}$ at $67^{\circ} \mathrm{C}$ in $6 \times \mathrm{SSC}$ containing $1 \times \mathrm{PM}[\mathrm{PM}$ is $0.02 \%(\mathrm{w} / \mathrm{v})$ bovine serum albumin, $0.02 \%(\mathrm{w} / \mathrm{v})$ Ficoll and $0.02 \%(\mathrm{w} / \mathrm{v})$ polyvinylpyrrolidone (Denhardt, 1966)|.

For the hybridization, nick-translated $\lambda$ prbt DNA (about $2 \times 10^{5}$ c.p.m. per track with a specific activity of about $5 \times 10^{7}$ c.p.m. $\mu \mathrm{g}^{-1}$ ) in 3 to $5 \mathrm{ml} 10 \mathrm{~mm}$-Tris $/ \mathrm{HCl}, 2 \mathrm{mM}$-EDTA, $1 \times \mathrm{PM}, 0.5 \%(\mathrm{w} / \mathrm{v})$ sodium dodecyl sulphate (SDS), $0.025 \%(\mathrm{w} / \mathrm{v})$ sonicated salmon sperm DNA (Calbiochem), $5 \times \mathrm{SSC}, \mathrm{pH} 7.5$ was sealed together with the nitrocellulose sheet in a polyethylene bag and incubated with gentle shaking in a water bath at $68{ }^{\circ} \mathrm{C}$ for $18 \mathrm{~h}$. The sheets were then washed twice for $30 \mathrm{~min}$ at $68^{\circ} \mathrm{C}$ in $200 \mathrm{ml}$ hybridization mixture from which all DNA was omitted, followed by a further 10 to 20 washes each for $30 \mathrm{~min}$ at $68{ }^{\circ} \mathrm{C}$ in $200 \mathrm{ml} 2 \times \mathrm{SSC}, 0.1 \%(\mathrm{w} / \mathrm{v})$ SDS, and a final wash in $2 \times \mathrm{SSC}$. After drying, the sheets were autoradiographed at $-70{ }^{\circ} \mathrm{C}$ using a tungstate intensifying screen and Kodak X-omat XH1 or Fuji RX film which had been subjected to a pre-exposure light flash (Laskey \& Mills, 1977).

\section{RES ULTS}

\section{Screening for rbt duplications}

The $K$. aerogenes evolvants were tested for the presence of $r b t$ duplications by using cloned $r b t$ DNA as a hybridization probe to identify fragments of restricted total bacterial DNA containing $r b t$ operon sequences. We used nick-translated DNA from the specialized lambda transducing phage $\lambda$ prbt (Neuberger \& Hartley, 1979) as the $r b t$ hybridization probe. The availability of phages $\lambda \mathrm{pr} b t$ and $\lambda \mathrm{p} r b t d a l$ [a phage derived from $\lambda \mathrm{p} r b t$ but which encodes the enzymes of both the ribitol operon and the closely linked D-arabitol operon (dal)] has enabled us to deduce a restriction cleavage map of the bacterial DNA in the region of the pentitol operons (Fig. $2 a$ ).

DNA extracted from the $K$. aerogenes evolvants superproducing ribitol dehydrogenase was digested with $B s t \mathbf{I}$, subjected to electrophoresis in agarose gels, transferred to nitrocellulose filter sheets by the method of Southern (1975) and hybridized with nick-translated $\lambda$ prbt DNA. The evolvants screened are indicated in the legend to Fig. 1 . The ancestral strain, strain $\mathrm{A}^{\prime}$, gave rise to three $\lambda \mathrm{p} r b t$-hybridizable $B s t \mathrm{I}$ fragments; their sizes were $6.7,1.65$ and $0.99 \mathrm{~kb}$ (Fig. $3 a$ ). As shown previously (Neuberger \& Hartley, 1979), the $6.7 \mathrm{~kb}$ Bst I fragment is generated by cleavage at Bst A and the BstI site on the chromosome to the left of $B s t \mathrm{~A}$. The $1.65 \mathrm{~kb}$ fragment is generated by cleavage at BstA and Bst $\mathrm{B}$; the weakness of this hybridization reflects the fact that $\lambda$ prbt contains only about 80 base pairs of homology with this fragment (T. Loviny, M. S. Neuberger \& B. S. Hartley, unpublished evidence). The $0.99 \mathrm{~kb}$ fragment is generated by cleavage at $B s t \mathrm{D}$ and $B s t \mathrm{E}$, the hybridization reflecting the existence of a repeated sequence in the region of the wild-type pentitol operons, as we have previously demonstrated (Neuberger \& Hartley, 1979). 

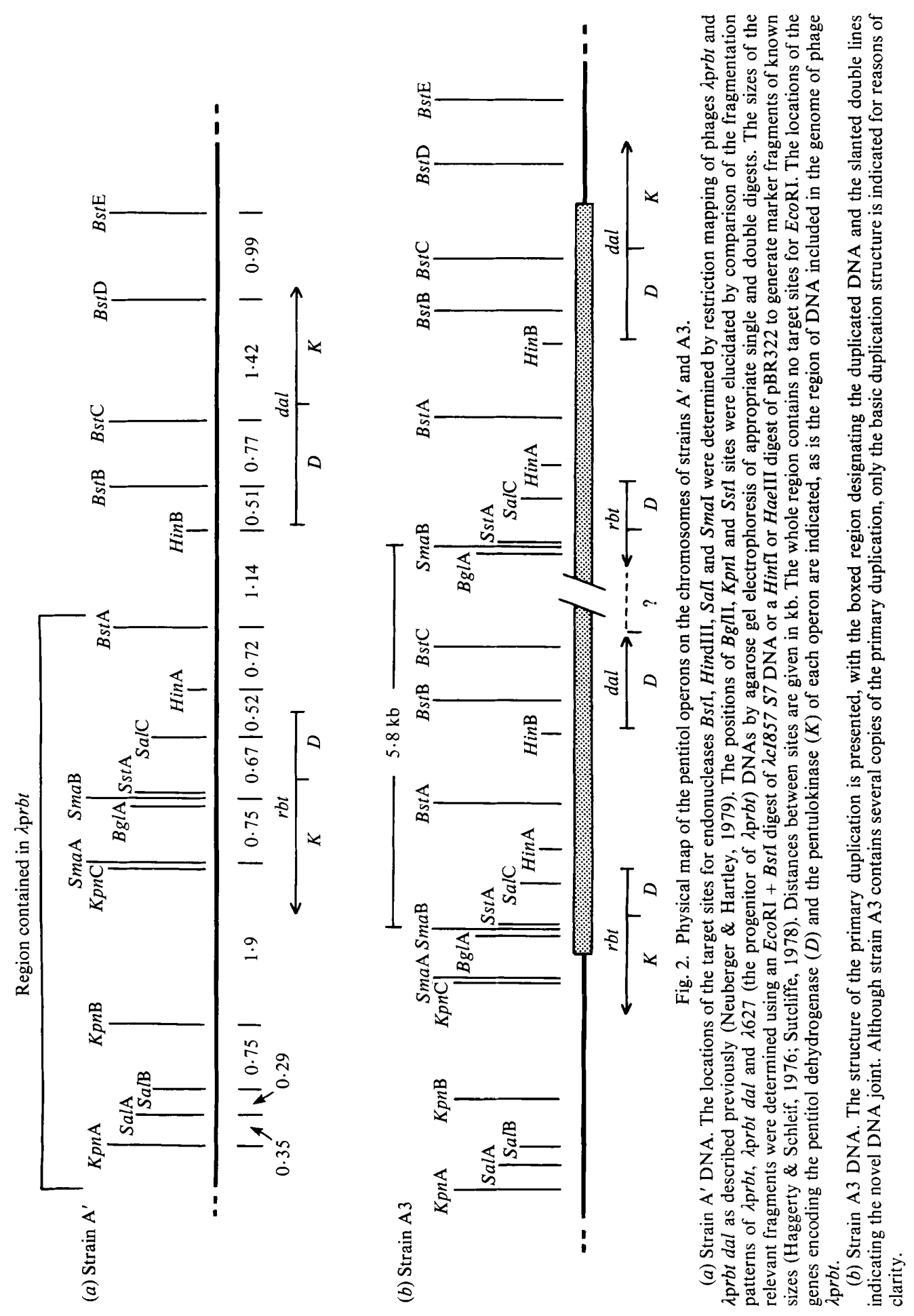
(a)

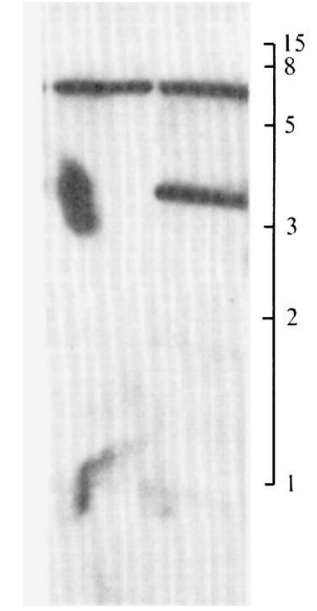

(c) $\quad(d) \quad(e) \quad(f) \quad(g)$

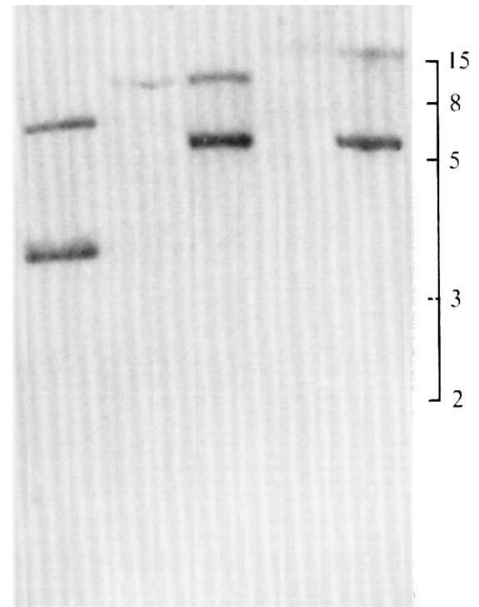

(h) (i) $(j)$

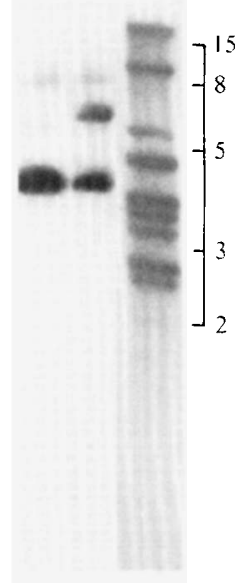

Fig. 3. Pattern of hybridization of $r b t$ DNA to DNA of strains $A^{\prime}$ and A3 digested with BstI, BglII, Sst I and Sall. Details are given in Methods. The scales indicate fragment sizes in kb. (a) Strain $\mathrm{A}^{\prime}$, BstI; (b) strain A3, BstI; (c) strain A3, BstI; $(d)$ strain A', BgIII; $(e)$ strain A3, BglII; $(f)$ strain $\mathrm{A}^{\prime}$, SstI; $(g)$ strain A3, SstI; (h) strain A', Sall: (i) strain A3, Sall; $(j) \lambda$ DNA, EcoRI + BstI.

Examination of the pattern of $\lambda$ prbt hybridization to BstI-digested DNA from the evolvant strains revealed that all except strain A3 gave an identical $B s t$ I hybridization pattern to that given by DNA from strain $\mathrm{A}^{\prime}$. This finding does not indicate that these evolvants do not harbour $r b t$ gene duplications. Our screening method would detect tandem direct gene duplications only if there were sequence homology between $\lambda$ prbt and the bacterial restriction fragment containing the novel DNA joint. Hence for strains other than A3, the duplications suggested by the genetic evidence (Rigby et al., 1974; Dothie, 1974) must span both the ribitol dehydrogenase $(r b t D)$ and D-ribulokinase $(r b t K)$ genes.

\section{Hybridization to BstI, SalI, SstI and BgIII digests of strain A3}

Strain A3 gave rise to a $\lambda$ prbt-hybridizable Bst 1 fragment of $3.4 \mathrm{~kb}$ in addition to the fragments of $6.7,1.65$ and $0.99 \mathrm{~kb}$ that were also found with wild-type $K$. aerogenes DNA (Fig. $3 a, b$ ). In order to glean more information about the basis of this altered $r b t$ fragmentation pattern, we compared the fragment hybridization patterns of DNA extracted from strains $\mathrm{A}^{\prime}$ and $\mathrm{A} 3$ after digestion with restriction endonucleases $S a l \mathrm{I}, S s t \mathrm{I}$ and $B g l \mathrm{II}$ (Fig. $3 d$ to $i$ ). With all three endonucleases, strain $\mathrm{A} 3$ gave rise to the same $\lambda \mathrm{p} r b t$ hybridizable fragments as strain $\mathrm{A}^{\prime}$ but in addition, with each endonuclease, strain $\mathrm{A} 3$ also gave rise to a new $r b t$ fragment of $5.8 \mathrm{~kb}$. This result is precisely what would be predicted if strain A3 contained a direct (rather than invert) duplication of $5.8 \mathrm{~kb}$ of DNA in the region of the $r b t$ operon, with the duplicated stretch of DNA containing a single target site for each of the three endonucleases. (It should be noted that, with such a structure, the same fragment hybridization pattern would be obtained with all three endonucleases whether the duplication were just a strict duplication or whether it were a triplication or higher amplification. We shall, however, unless otherwise specified, use the term duplication loosely to include the primary duplication and higher amplifications.) In order to explain why the novel $B s t \mathrm{I}$ fragment in strain A3 is only $3.4 \mathrm{~kb}$ in length (rather than $5.8 \mathrm{~kb}$ ) it is necessary to postulate that the duplicated DNA contains more than one target site for Bst I. Given the BstI cleavage map of the wild-type pentitol operons (Fig. $2 a$ ), it is clear that the only structure that 


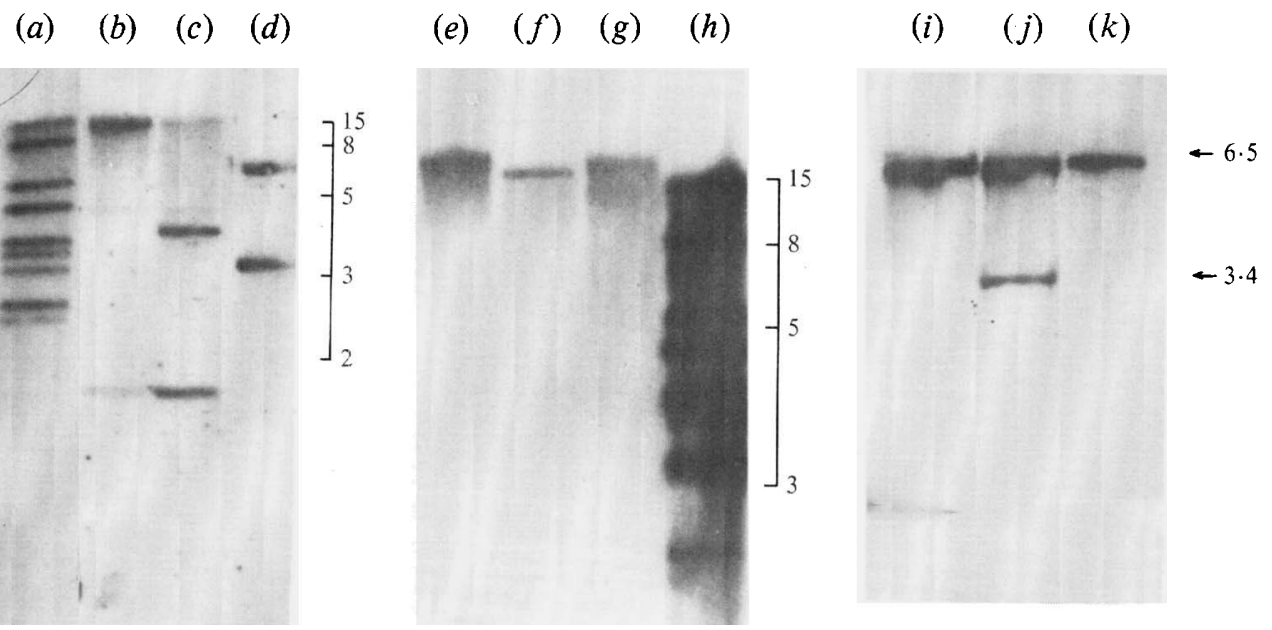

Fig. 4. Pattern of hybridization of $r b t$ DNA to DNA of strains $\mathrm{A}^{\prime}, \mathrm{A} 3$ and A3S5 digested with HindIII, $E c o$ RI and BstI. Details are given in Methods. The scales indicate fragment sizes in kb. (a) $\lambda$ DNA $E c o$ RI + BstI; (b) strain A',HindIII; (c) strain A3, HindIII; (d) strain A3, BstI; (e) strain A3, Eco RI; $(f)$ strain $\mathrm{A}^{\prime}, E$ coRI; $(g)$ strain A3, EcoRI; $(h) \lambda$ DNA, EcoRI + BstI; $(i)$ strain A', BstI; $(j)$ strain A3, Bst $\mathrm{I} ;(k)$ strain A3S5, BstI.

will fit the data is a duplication containing BstI sites Bst A, Bst $\mathrm{B}$ and Bst $\mathrm{C}$. The new BstI fragment then extends from $B s t \mathrm{C}$ across the novel joint to $B s t \mathrm{~A}$ (Fig. $2 b$ ), so its expected size would be $5.8 \mathrm{~kb}$ minus the BstA-BstC distance $(2.42 \mathrm{~kb})$, i.e. $3.4 \mathrm{~kb}$, as observed in Fig. $3(b, c)$.

\section{Hybridization to a HindIII digest of strain A3}

If the proposed structure of the strain A3 duplication is correct, then the duplication contains two HindIII sites separated by $1.8 \mathrm{~kb}$. The size of the rbt hybridizable fragment peculiar to strain A3 should therefore be $5.8 \mathrm{~kb}$ minus $1.8 \mathrm{~kb}$, i.e. $4.0 \mathrm{~kb}$. Hybridization of nick-translated $\lambda$ prbt DNA to HindIII digests of DNA from strains $\mathrm{A}^{\prime}$ and A3 confirmed that this was indeed the case (Fig. $4 b, c$ ).

\section{Hybridization to an EcoRI digest of strain $A 3$}

It is predicted from the duplication structure presented in Fig. $2(b)$ that the A3 duplication contains no site for EcoRI; furthermore, there are no EcoRI sites on the bacterial DNA substitution of phage $\lambda \mathrm{p} r b t$. Therefore, $\lambda \mathrm{p} r b t \mathrm{DNA}$ is expected to hybridize to a single $E c o \mathrm{RI}$ fragment of strain $\mathrm{A}^{\prime}$ DNA and to a single, but larger, EcoRI fragment of strain A3 DNA; this was shown to be the case (Fig. $4 e, f, g$ ). It should be possible to deduce the number of copies of the $r b t$ duplication present in strain A3 from the difference in size of the strain A3 and strain $\mathrm{A}^{\prime}$ rbt EcoRI fragments. However, owing to the largeness of the fragments, we have not been able to deduce their sizes with any accuracy from their mobilities in agarose gel electrophoresis. Rough estimates of $20 \mathrm{~kb}$ for the strain $\mathrm{A}^{\prime}$ rbt EcoRI fragment and $45 \mathrm{~kb}$ for the strain A3 rbt EcoRI fragment were obtained by electrophoresis in $0.4 \%$ agarose gels for long periods using unrestricted DNA from wild-type $\lambda$ and unrestricted and EcoRI-digested DNA from the $\lambda$ deletion mutant, $\lambda 627$ (Neuberger \& Hartley, 1979) as size markers. This is consistent with there being four or five copies of the duplicated DNA tandemly arranged on the strain A3 chromosome. However, given the instability of the A3 duplication (see below), the cells in a culture of strain A3 are not expected to be homogeneous with respect to $r b t$ gene dosage. 
Table 1. Activities of pentitol catabolic enzymes in strains $A^{\prime}, A 3$ and derivatives

Dehydrogenase activities are expressed as units $(\mathrm{mg} \text { soluble protein })^{-1}$ and D-ribulokinase activities are expressed as the decrease in $A_{560}$ units $\min ^{-1}$ (mg protein $)^{-1}$. Strains A3S2. A3S3. A3S4 and A3S5 are slow-growing segregants of strain A3.

\begin{tabular}{lccc}
\multicolumn{1}{c}{ Strain } & $\begin{array}{c}\text { Constitutive } \\
\text { ribitol dehydrogenase }\end{array}$ & $\begin{array}{c}\text { Constitutive } \\
\text { D-ribulokinase }\end{array}$ & $\begin{array}{c}\text { Induced } \\
\text { D-arabitol dehydrogenase }\end{array}$ \\
A $^{\prime}$ & $2 \cdot 1$ & $0 \cdot 36$ & $0 \cdot 16$ \\
A3 & 13 & $0 \cdot 38$ & $1 \cdot 1$ \\
A211 & $5 \cdot 3^{*}$ & $2 \cdot 4$ & $\mathrm{ND}$ \\
A3S5 & $2 \cdot 0$ & $0 \cdot 43$ & $0 \cdot 19$ \\
A3S2, A3S3, A3S4 & $1 \cdot 9,1 \cdot 8,2 \cdot 0$ & $\mathrm{ND}$ & $\mathrm{ND}$
\end{tabular}

ND, Not determined.

* The ribitol dehydrogenase specific activity of strain A211 is considerably lower than that determined previously (Rigby et al., 1974); however, we have frequently observed a considerable decrease in the specific activity in extracts of enzyme superproducers following prolonged storage of the strains.

\section{Hybridization to SmaI and KpnI digests of strain A3}

In order to locate the left-hand end of the duplication more precisely, we looked at the $\lambda$ prbt fragment hybridization pattern obtained after digestion with SmaI. From the data presented so far, it is expected that the A3 duplication should extend through restriction target $S m a$ B, but the data do not allow us to deduce whether the duplication also extends through $S m a$ A. The results of a fragment hybridization experiment (not shown) showed that the SmaI rbt fragment peculiar to strain A3 is $5.8 \mathrm{~kb}$, thus indicating that the duplication contains only a single SmaI site - which is presumably SmaB. Similarly, comparison of the $\lambda$ prbt Kpnl fragment hybridization patterns of DNA from strains $\mathrm{A}^{\prime}$ and A3 indicates that the duplicated DNA contains no sites for $K p n I$; this is consistent with the left-hand end of the A3 duplication lying somewhere between $S m a$ B and $S m a$ A.

\section{Instability of the A3 duplication}

Strains containing tandem gene duplications are expected to segregate at high frequency haploid derivatives generated by homologous recombination between the copies of the repeated DNA. In the case of tandem gene duplications containing $r b t D$, segregants can be recognized by virtue of their lower rate of growth on xylitol. As described in Methods, strain A3 gave rise to derivatives growing more slowly on xylitol at a frequency of $10^{-3}$. Whereas the specific activity of ribitol dehydrogenase in strain A3 was 13 units (mg soluble protein $)^{-1}$, the activity of this enzyme in four segregants examined was approximately the same as that in strain $\mathrm{A}^{\prime}$ (Table 1). (The only colonies picked were those growing considerably more slowly on minimal xylitol plates; segregants possessing intermediate levels of ribitol dehydrogenase activity would therefore probably not have been detected.) If these segregants had arisen by recombinational 'looping out' of the duplication, the A3 segregants would be expected to give wild-type $\lambda$ p $r b t$ fragment hybridization patterns. DNA extracted from one of the segregants, strain A3S5, was indeed found to have lost the A3-characteristic $3.4 \mathrm{~kb} \mathrm{BstI} \mathrm{rbt}$ fragment and its fragment hybridization pattern was indistinguishable from that of strain $\mathrm{A}^{\prime}$ (Fig. $4 i, j$, $k$ ). Thus, from the point of view of both DNA structure and ribitol dehydrogenase specific activity, segregant A3S5 is indistinguishable from the ancestral strain, strain $A^{\prime}$. Therefore, the elevated ribitol dehydrogenase activity in strain A3 is likely to be solely due to gene amplification.

\section{Correlation of the physical and genetic structure of the A3 duplication}

Comparison of the activities of the pentitol catabolic enzymes in strains $A^{\prime}$ and A3 (Table 1) shows that both constitutive ribitol dehydrogenase activity and induced D-arabitol 
dehydrogenase activity are about six times higher in strain A3 than in strain $\mathrm{A}^{\prime}$. Interestingly, however, strains $\mathrm{A}^{\prime}$ and $\mathrm{A} 3$ have similar constitutive D-ribulokinase activities. As expression of $r b t D$ and $r b t K$ is coordinately controlled in the wild-type operons (Charnetzky \& Mortlock, 1974), one would expect that evolvants containing duplications of the entire rbt operon should have elevated activities of both ribitol dehydrogenase and D-ribulokinase. This prediction is supported by the observation that evolvant A211, which is believed to contain a duplication of the entire rbt operon (see Fig. 1), shows elevated activities of both ribitol dehydrogenase and D-ribulokinase (Table 1). Thus, the fact that D-ribulokinase synthesis is not amplified in evolvant $\mathrm{A} 3$ suggests that the $\mathrm{A} 3$ duplication includes $r b t D$ but not an intact $r b t K$. Similarly, the increased D-arabitol dehydrogenase activity in evolvant A3 suggests that the D-arabitol dehydrogenase structural gene (dalD) is included in the A3 duplication.

Clearly, however, whilst the enzyme specific activities of evolvant A3 are consistent with it harbouring a rbtD-dalD duplication, other types of mutations could be invoked to explain these enzyme activities. More convincing evidence for the genetic structure of the duplication can be obtained by an accurate correlation of the physical and genetic maps of the wild-type pentitol operons.

We have previously presented evidence for the approximate locations of the $r b t$ and $d a l$ structural gene transcriptions, which was obtained by hybridizing in vivo labelled messenger RNA to $\lambda \mathrm{p} r b t$ and $\lambda \mathrm{p} r b t d a l$ restriction fragments (Neuberger \& Hartley, 1979). However, current work on the DNA sequence analysis of the pentitol operons (T. Loviny, T. J. Knott \& B. S. Hartley, unpublished evidence) allows us to locate the coding sequences more accurately. Comparison of the amino acid sequence of ribitol dehydrogenase (Morris et al., 1974 ) with the DNA sequence in the region of endonuclease site SalC shows that site $\mathrm{SalC}$ is located within rbtD, cleavage taking place at position 68 in the amino acid sequence. Knowing that the D-ribulokinase gene is located promoter-distal to rbtD (Charnetzky \& Mortlock, 1974), that the sub-unit molecular weights of ribitol dehydrogenase and D-ribulokinase are 27000 and 60000, respectively (Taylor et al., 1974; Neuberger et al., 1980) and assuming that there is no extensive overlap between the ribitol dehydrogenase and D-ribulokinase coding sequences, it is possible to state that, whereas $r b t D$ starts between $H i n \mathrm{~A}$ and $S a l \mathrm{C}$ and terminates to the right of $S m a \mathrm{~B}, r b t K$ must terminate at least 700 base pairs to the left of SmaA. Thus, this analysis demonstrates that the A3 duplication is expected to contain an intact $r b t D$ but not an intact $r b t K$.

Similarly, comparison of the DNA sequence in the region of endonuclease site $B s t C$ with the sequences of peptides derived from D-arabitol dehydrogenase shows that dalD begins just to the right of $\operatorname{Hin} \mathrm{B}$ and terminates about 200 base pairs to the right of BstC (T. J. Knott \& B. S. Hartley, unpublished evidence). The fragment hybridization analysis showed that the right-hand end of the A 3 duplication is located somewhere between $B s t \mathrm{C}$ and $B s t \mathrm{D}$. From the size of the duplication, it is possible to state that, if the duplication contains only a single novel DNA joint (see Discussion), its right-hand end must lie at least 700 base pairs to the right of $B s t$ C. Thus, it is probable that the A3 duplication includes an intact dalD gene, a prediction supported by the D-arabitol dehydrogenase specific activity.

\section{Extended restriction map of the wild-type pentitol operons}

The restriction map of the wild-type pentitol operon DNA presented in Fig. 2(a) was deduced from an analysis of phages $\lambda \mathrm{p} r b t$ and $\lambda \mathrm{prbt}$ dal. However, an analysis of the $r b t$ fragment hybridization pattern of DNA extracted from strain $\mathrm{A}^{\prime}$ enables us to confirm that the structure of the pentitol operon DNA on the transducing phages is identical to that on the $K$. aerogenes chromosome. Hybridization of $\lambda p r b t$ DNA to restriction digests of strain $\mathrm{A}^{\prime}$ DNA reveals, in addition to other fragments, a $4.0 \mathrm{~kb} S a l \mathrm{l}$ fragment (Fig. $3 h$ ), a $1.8 \mathrm{~kb}$ HindIII fragment (Fig. $4 b$ ), as well as 1.9 and $1.4 \mathrm{~kb} \mathrm{KpnI} \mathrm{fragments} \mathrm{and} \mathrm{a} 0.75 \mathrm{~kb}$ SmaI fragment (data not shown), as would be predicted from the map shown in Fig. 2(a). However, it is possible, on the basis of the chromosomal fragment hybridization patterns, to 


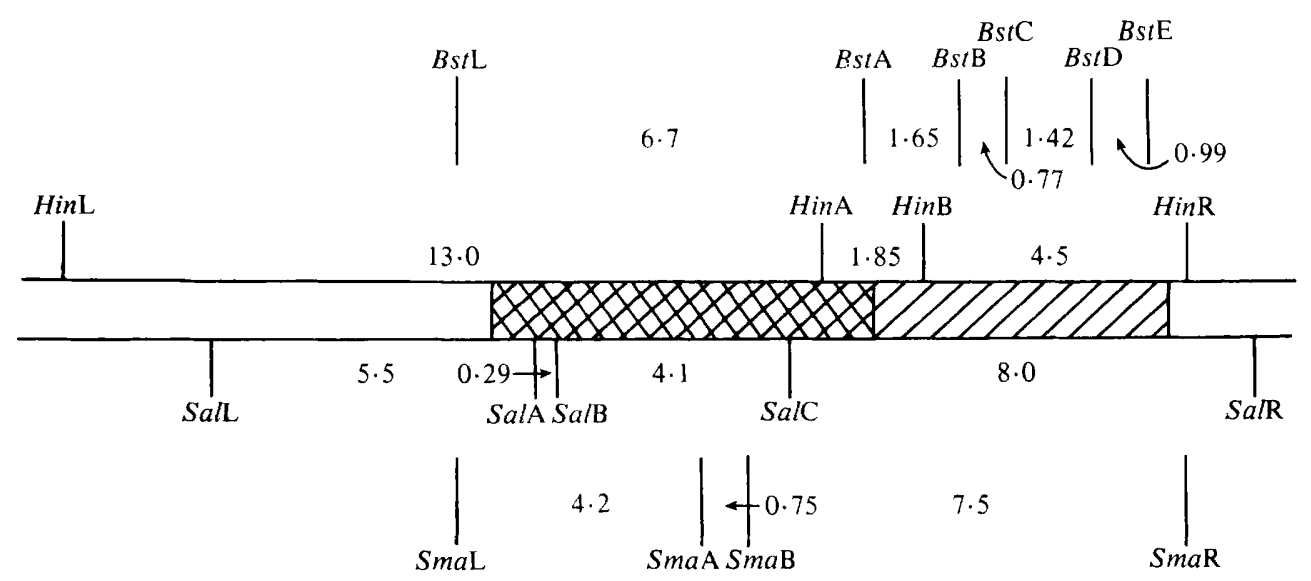

Fig. 5. Restriction map of the chromosomal environment of the pentitol operons. The whole shaded region shows the bacterial insert in $\lambda$ prbt dal and the cross-hatched region the insert in $\lambda$ prbt. The restriction sites $H i n \mathrm{~L}, S a l \mathrm{~L}$ etc. are those closest to these regions on the left and $H i n \mathrm{R}, S a / \mathrm{R}$ etc. indicate neighbouring sites on the right. Sizes of restriction fragments are in $\mathrm{kb}$. The data do not allow ordering of $S m a \mathrm{~L}$ and Bst $\mathrm{L}$ or $\operatorname{Sma\mathrm {R}}$ and $\operatorname{Hin} \mathrm{R}$ and the assigned location of Hin $\mathrm{R}$ is tentative (see text).

deduce an extended cleavage map of the DNA of the pentitol operons - including DNA on the bacterial chromosome adjacent to the region cloned in phage $\lambda$ prbt dal (Fig. 5). Thus, as shown previously (Neuberger \& Hartley, 1979), there is a BstI site on the $K$. aerogenes chromosome $6.7 \mathrm{~kb}$ to the left of $B s t \mathrm{~A}$. Similarly, examination of the fragment hybridization pattern obtained with other endonucleases reveals $S a l \mathrm{I}$ sites $5.5 \mathrm{~kb}$ to the left of $S a l \mathrm{~A}$ and $8 \mathrm{~kb}$ to the right of $S a l \mathrm{C}$, and $S m a I$ sites $4.2 \mathrm{~kb}$ to the left of $S m a \mathrm{~A}$ and $7.5 \mathrm{~kb}$ to the right of SmaB. The HindIII site to the left of HinA is located about $13 \mathrm{~kb}$ away and weak hybridization of $\lambda$ prbt DNA to a $4.5 \mathrm{~kb}$ HindIII fragment of strain $\mathrm{A}^{\prime}$ DNA is also seen (Fig. $4 b$ ). This probably indicates that the chromosomal HindIII site to the right of $\operatorname{Hin} \mathrm{B}$ is $4.5 \mathrm{~kb}$ away - the hybridization being due to the repeated sequence discussed earlier. However, it is notable that strain $\mathrm{A}^{\prime}$ appears to give rise to only one $\lambda$ prbt-hybridizable $S s t$ I fragment (15 kb) and only one $B g l \mathrm{II}$ fragment ( $10 \mathrm{~kb}$ ); each enzyme would be expected to give rise to two chromosomal $\lambda$ prbt-hybridizable fragments. We believe this discrepancy is best explained by the suggestion that, with each endonuclease, the single radioactive bands seen in the autoradiographs are caused by hybridization to two fragments of similar electrophoretic mobilities which have not been resolved.

\section{I S C US S I ON}

The fragment hybridization technique for screening for gene duplications has the major limitation that duplications will only be detected if the restriction fragment containing the novel DNA joint also contains sequence homology with the probe DNA. The advantage of the approach lies in the fact that it is not necessary to clone the duplication and that it can yield a considerable amount of information about the structure and location of some duplications - information which, in many cases, would not be readily gleaned from genetic analysis.

Evolvant A3 most probably contains about five copies of $r b t D$ clustered together on the chromosome. As shown in Fig. 2(b), the fragment hybridization analysis suggests a tandemly repeated structure of a $5.8 \mathrm{~kb}$ segment of DNA which encodes ribitol dehydrogenase and 
D-arabitol dehydrogenase, but not a full-length D-ribulokinase. Our data cannot entirely exclude a repeating structure in which the duplicated DNA segments are separated by extraneous 'spacer DNA', which could be up to $1.3 \mathrm{~kb}$ in length. The size of the primary duplication would then be $5.8 \mathrm{~kb}$ minus the length of the spacer DNA segment. However, such a structure would require two novel DNA joints and the spacer region could contain no target sites for endonucleases EcoRI, BstI, HindIII, Sall, SstI, BglII, SmaI or KpnI. For this reason, and also by analogy with duplications isolated in other experimental systems (Emmons \& Thomas, 1977; Charlier et al., 1979), we prefer the strictly tandem duplication structure, containing no spacer region.

The upper limit placed on the size of the possible spacer could be reduced by screening with more endonucleases. Electron microscopic analysis of the cloned duplication region could reduce the uncertainty to 100 to 200 base pairs (Charlier et al., 1979), but ultimately DNA sequence analysis will be necessary to establish that the duplications are directly tandem. As a general method for rapid cloning of the various direct $r b t$ duplications it should be possible to use in vivo recombination between the duplicated bacterial rbt genes and a plasmid containing part of $r b t D$; recombinant plasmids containing the novel DNA joints of the $r b t$ duplications could then be distinguished as they would contain intact $r b t D$ genes and thus confer the ability to grow on xylitol.

If the duplication in evolvant A3 is strictly tandem, it would be of interest to know whether transcription initiated at the $r b t$ promoter continues across the novel DNA joint to synthesize 'anti-dal' messenger RNA and, similarly, whether induction of the dal operon results in the formation of anti-rbt RNA, as hinted in Fig. $2(b)$. (Although growth in the presence of $0.2 \%(\mathrm{w} / \mathrm{v}) \mathrm{D}$-arabitol results in a $30 \%$ drop in the ribitol dehydrogenase specific activity of strain $\mathrm{A} 3$, a similar drop is seen with strain $\mathrm{A}^{\prime}$ and is probably due to catabolite repression.]

We have previously demonstrated the existence of a repeated sequence in the region of the pentitol operons (Neuberger \& Hartley, 1979). A sequence present in the SalB-SalC fragment is also present in $B s t \mathrm{D}-B s t \mathrm{E}$, but it is not known whether the copies of the repeat are oriented in the same or opposite directions. However, the location of the repeat precludes its being implicated in the formation of the A3 duplication. If the A3 duplication is strictly tandem, it was formed by recombination between a site lying between $S m a \mathrm{~A}$ and $S m a \mathrm{~B}$ and a site lying between $B s t C$ and $B s t \mathrm{D}$. DNA sequence analysis will reveal whether a short stretch of sequence homology provided a focus for such recombination.

We thank Rosemary Patterson for expert technical assistance, Audrey Sibley for the preparation of the manuscript, Thérèse Loviny and Tim Knott for allowing us to present deductions based on their unpublished DNA sequence data and Peter David for helpful criticisms of the manuscript. The work was supported by a grant from the Science Research Council.

\section{REFERENCES}

Anderson, R. P. \& Roth, J. (1977). Tandem genetic duplications in phage and bacteria. Annual Review of Microbiology 31, 473-506.

ANDERson, R. P. \& Roth, J. (1978). Tandem genetic duplication in Salmonella typhimurium: amplification of the histidine operon. Journal of Molecular Biology 126, 53-71.

Beeftinck, F., Cunin, R. \& Glansdorff, N. (1974). Arginine gene duplication in recombination proficient and deficient strains of Escherichia coli. Molecular and General Genetics 161, 185-196.
Bergquist, P. L. \& Jamieson, A. J. (1977). Genetic inversion in the formation of an Hfr strain from a temperature-sensitive $\mathrm{F}^{\prime}$ gal strain. Journal of Bacteriology 129, 282-290.

Charlier, D., Crabeel, M., Cunin, R. \& GlansDorfF, N. (1979). Tandem and inverted repeats of the arginine genes in Escherichia coli. Structural and evolutionary considerations. Molecular and General Genetics 174, 75-88.

Charnetzky, W. T. \& Mortlock, R. P. (1974). Close genetic linkage of the determinants of the 
ribitol and D-arabitol catabolic pathways in Klebsiella aerogenes. Journal of Bacteriology 119 , 176-182.

DenhaRDT, D. T. (1966). A membrane-filter technique for the detection of complementary DNA. Biochemical and Biophysical Research Communications 23, 641-646.

DOTHIE, J. M. (1974). The evolution of ribitol dehydrogenase in Klebsiella aerogenes. Ph.D. thesis, University of Cambridge.

Emmons, S. W. \& Thomas, J. O. (1975). Tandem genetic duplications in phage $\lambda$ : the locations of spontaneously arising duplications. Journal of Molecular Biology 91, 147-152.

Greene, P. J., Betlach, M. C., Goodman, H. M. \& BOYER, H. W. (1974). The EcoRI restriction endonuclease. Methods in Molecular Biology 7, 88-112.

HAGgerTy, D. M. \& Schleif, R. F. (1976). Locations on phage lambda of the cleavage sites of the site-specific endonuclease from Bacillus amyloliquefaciens H. Journal of Virology 18, 659-663.

Hartley, B. S., Altosaar, I., Dothie, J. M. \& Neuberger, M. S. (1976). Experimental evolution of a xylitol dehydrogenase. In Structure-Function Relationships in Proteins, pp. 191-200. Edited by R. Markham \& R. W. Horne. Amsterdam: North Holland.

Hill, C. W., Grafstrom, R. H., Harnish, B. W. \& Hillman, B. S. (1977). Tandem duplications resulting from recombination between the ribosomal RNA genes in Escherichia coli. Journal of Molecular Biology 116, 407-428.

Laskey, R. A. \& Mills, A. D. (1977). Enhanced autoradiographic detection of ${ }^{32} \mathrm{P}$ and ${ }^{125} \mathrm{I}$ using intensifying screens and hypersensitized film. FEBS Letters 82, 314-316.

LEwIS, E. B. (1951). Pseudoallelism and gene evolution. Cold Spring Harbor Symposia on Quantitative Biology 16, 159-174.

MARMUR, J. (1961). A procedure for the isolation of DNA from microorganisms. Journal of Molecular Biology 3, 208-218.

Miller, G. L. (1959). Protein determination for large numbers of samples. Analytical Chemistry 31, 964.

Morris, H. R., Williams, D. H., Midwinter, G. G. \& HARTley, B. S. (1974). A mass spectrometric sequence study of the enzyme ribitol dehydrogenase from Klebsiella aerogenes. Biochemical Journal 141, 701-713.
Neuberger, M. S. \& Hartley, B. S. (1979). Investigations into the Klebsiella aerogenes pentitol operons using specialised transducing phages $\lambda \mathrm{pr} b t$ and $\lambda$ prbt dal. Journal of Molecular Biology 132, 435-470.

Neuberger, M. S., Patterson, R. A. \& Hartley, B. S. (1979). Purification and properties of Klebsiella aerogenes $\mathrm{D}$-arabitol dehydrogenase. Biochemical Journal 183, 31-42.

Neuberger, M. S., Hartley, B. S. \& Walker, J. E. (1980). Purification and properties of D-ribulokinase and D-xylulokinase from Klebsiella aerogenes. Biochemical Journal (in the Press).

OHNo, S. (1970). Evolution by Gene Duplication. Berlin, Heidelberg \& New York: Springer.

Rigby, P. W. J., Burleigh, B. D. \& Hartley, B. S. (1974). Gene duplication in experimental enzyme evolution. Nature, London 25 1, 200-204.

Riley, M., Solomon, L. \& ZipKas, D. (1978). Relationship between gene function and gene location in Escherichia coli. Journal of Molecular Evolution 11, 47-56.

Smithies, O., Connell, G. E. \& Dixon, G. H. (1962). Chromosomal rearrangements and the evolution of the haptoglobin genes. Nature, London 196, 232236.

SOUTHERN, E. M. (1975). Detection of specific sequences among DNA fragments separated by gel electrophoresis. Journal of Molecular Biology $\mathbf{9 8 ,}$ 503-517.

Straus, D. S. \& Hoffmann, G. R. (1975). Selection for a large genetic duplication in Salmonella typhimurium. Genetics 30, 227-237.

SUTClifFE, J. G. (1978). pBR322 restriction map derived from the DNA sequence: accurate DNA size markers up to 4361 nucleotide pairs long. Nucleic Acids Research 5, 2721-2728.

TAylor, S. S., Rigby, P. W. J. \& Hartley, B. S. (1974). Ribitol dehydrogenase from Klebsiella aerogenes: purification and subunit structure. Biochemical Journal 141, 693-700.

Trowsdale, J. \& Anagnostopoulos, C. (1975). Evidence for the translocation of a chromosome segment in Bacillus subtilis strains carrying the trpE26 mutation. Journal of Bacteriology 122 , 886-898.

Wu, T. T., Lin, E. C. C. \& Tanaka, S. (1968). Mutants of Aerobacter aerogenes capable of utilizing xylitol as a novel carbon source. Journal of Bacteriology 96, 447-456. 